\title{
Caracterización de soldaduras circunferenciales de aceros avanzados 9Cr: Parte 1
}

\author{
(Caracterização de soldas circunferências de aços avançados 9Cr: Parte 1) \\ (Characterization of circumferential welds of 9Cr advanced steels: Part 1)
}

\author{
Mabel Ramini ${ }^{1}$, Estela Surian ${ }^{1,2}$, María José Castillo ${ }^{1}$ y Mónica Zalazar ${ }^{3}$ \\ ${ }^{1}$ Universidad Tecnológica Nacional, Facultad Regional San Nicolás/DEYTEMA, San Nicolás, Argentina. mramini@frsn.utn.edu.ar \\ ${ }^{2}$ Universidad Nacional de Lomas de Zamora, Facultad de Ingeniería/Secretaría de Investigación, Lomas de Zamora, Argentina. \\ esurian@arnet.com.ar \\ ${ }^{3}$ Universidad Nacional del Comahue, Facultad de Ingeniería, Neuquén, Argentina. monica.zalazar@fain.uncoma.edu.ar
}

\begin{abstract}
Resumo
Os aços 9\%Cr são amplamente utilizados à alta temperatura, principalmente em aplicações relacionadas à geração de energia. Ultimamente têm sido desenvolvidas variantes desses materiais incorporando B, W e Co, entre outros elementos, ao sistema de liga. Devido à escassez de informação sobre a influência do procedimento de soldagem sobre a junta soldada, neste trabalho se estudou o comportamento do metal base e do metal de solda em juntas circunferências soldadas com dois consumíveis diferentes pelo processo de soldagem semi-automático $\mathrm{FCAW}$, sob proteção gasosa de $\mathrm{Ar} / 20 \% \mathrm{CO}_{2}$. Realizaram-se tratamentos térmicos pós-soldagem a $760^{\circ} \mathrm{C}$ durante 4 e 2 horas. Utilizaram-se dois arames tubulares de escória rutílica, ligados ao 9Cr1Mo e 9Cr0,5Mo1WCo. Analisou-se a microestrutura nas zonas do metal de solda, afetada pelo calor (zonas de grão grosso, grão fino, intercrítica e subcrítica) e metal base. Determinaram-se a composição química do metal de solda e as propriedades de tração transversal da junta, e realizaram levantamento do perfil de microdureza. Em todos os casos se observaram valores máximos de dureza na zona correspondente ao metal de soldadura e uma diminuição na zona afetada pelo calor desde a zona de grão grosso até o metal base, obtendo se valores mínimos na zona intercrítica, inferiores aos correspondentes ao aço base. Tanto a dureza como a resistência à tração aumentaram quando foi utilizado o arame $9 \mathrm{Cr} 0,5 \mathrm{Mo} 1 \mathrm{~W}$. O tratamento térmico mais curto permitiu alcançar maior resistência em todos os casos. Numa próxima etapa se fará as medições de tenacidade e de resistência ao creep.
\end{abstract}

Palavras-chave: Aço 9Cr; Tratamento térmico pós-soldagem; Microestrutura; Junta soldada; Arames tubulares.

\begin{abstract}
Cr steels are widely used for high temperature service, principally in applications related to energy generation. Lately several variants of these materials have been developed through the addition of $\mathrm{B}, \mathrm{W}$ and Co, among others. Due to the fact that information on influence of welding procedure on the welded joint is scarce, the objective of this work was to study the behavior of both the base metal and the weld metal of circumferential weldments performed using semiautomatic welding process under gas mixture protection. Two FCAW rutilic slag wires, alloyed with $9 \mathrm{Cr} 1 \mathrm{Mo}$ and $9 \mathrm{Cr} 0.5 \mathrm{Mo} 1 \mathrm{~W} 1 \mathrm{Co}$ respectively, were used under $\mathrm{Ar} / 20 \% \mathrm{CO}$, gas shielding. Post weld heat treatments of 4 and 2 hours have been carried out. The microstructure was analyzed in the different zones: weld metal, heat affected (coarse and fine grain, intercritical and subcritical) and not affected base metal. Chemical composition of the weld metal, transversal tensile properties of the joint and microhardness along the weld transversal section were determined. In all cases it was obtained maximum hardness values in the weld metal and a decrease in this property in the heat affected zone from the coarse grain zone to the base metal one. The minimal hardness values were achieved in the intercritical zone, lower than those of the metal base. Hardness and tensile strength increased when the weldment was performed with the $9 \mathrm{Cr} 0,5 \mathrm{Mo} 1 \mathrm{~W}$ wire. The shorter heat treatment allowed obtaining higher values of tensile strength, in all the cases. Toughness and creep resistance determinations will be the next step.
\end{abstract}

Key-words: 9Cr steel; Post welding heat treatment; Microstructure; Welded joint; Tubular wire

\section{Introducción}

Dentro de la familia de aceros ferríticos resistentes al creep (CSEFS-creep strength-enhanced ferritic steel) se encuentran los

Recebido em 11/12/2013, texto final em 19/02/2014. aceros ASTM 335 Gr P91, ampliamente utilizados en reemplazo de aleaciones tradicionales Cr-Mo. En general, el estado del arte de este tema reporta pocos problemas acerca de su soldabilidad; sin embargo, suelen encontrarse fallas prematuras debido a un mal diseño de la soldadura. Procedimientos incorrectos y tratamientos térmicos inadecuados suelen ser los responsables de estas fallas [1]. Las mayores causas de falla en uniones soldadas de los CSEFS se deben a:

- Formación de ferrita delta 
- Segregación de impurezas

- Crecimiento excesivo del grano austenítico primario

- Formación de una zona intercrítica debido al enfriamiento desde la zona de estructura dúplex (austenítico-ferrítica)

Además de estas heterogeneidades microestructurales la soldadura genera microrregiones que a su vez difieren en su composición química. Estas modificaciones microestructurales y microquímicas tienen lugar en volúmenes muy pequeños dentro de la zona fundida (ZF) y en la zona afectada por el calor (ZAC) [2]. Los CSEFS poseen una microestructura martensítica y/o bainítica con carburos y/o carbonitruros estables a altas temperaturas. Para mantener las condiciones que aseguren un buen comportamiento al creep, tanto el ciclo térmico de la soldadura como el tratamiento térmico post soldadura (PWHT) deben ser controlados.

El rango de temperaturas propuesto para el PWHT requiere no superar la temperatura crítica $\mathrm{Ac}_{1}$, la cual está definida por la composición química del metal de soldadura (integrado por el metal base fundido y el aporte del consumible), especialmente por el contenido de $\mathrm{Ni}+\mathrm{Mn}$. Por otro lado se recomienda seleccionar la mayor temperatura posible $\left(10 / 20^{\circ} \mathrm{C}<\mathrm{Ac}_{1}\right)$ teniendo en cuenta que para grandes piezas el gradiente térmico entre la superficie y el interior suele ser importante [1].

En la ZAC del metal base (MB) de una junta soldada ocurren diferentes transformaciones según el ciclo térmico a que estuvo sometida cada zona respecto de la línea de fusión (LF). Las temperaturas alcanzadas en cada zona pueden relacionarse con el diagrama de equilibrio para los aceros P91 que se muestra en la Figura 1, donde se definen las diferentes zonas y subzonas correspondientes a una unión soldada: zona fundida o metal de soldadura (MS), zonas afectadas por el calor de grano grueso (ZACGG), de grano fino (ZACGF), intercrítica (ZACIC) y subcrítica (ZACSC). En la ZACIC es donde generalmente ocurren las fallas por creep. Este tipo de falla se conoce como Tipo IV [3]. En las zonas afectadas por altas temperaturas se produce un crecimiento del grano austenítico primario, lo que resulta en una reducción en la tenacidad de la junta. Cuando se supera la temperatura $\mathrm{Ac}_{4}$, en los bordes de los granos austeníticos primarios comienza a nuclear ferrita delta, frenando en cierta medida el crecimiento de los mismos; la menor solubilidad en la ferrita del $\mathrm{C}$ y otros elementos estabilizadores de la austenita, resulta en la difusión de estos elementos hacia la austenita; la ferrita delta se enriquece en elementos alfágenos, como el cromo. Esta diferencia en composición química, en el enfriamiento, puede generar transformación incompleta dando por resultado una estructura martensítica con ferrita retenida en la ZACGG [4]. Pequeñas cantidades de ferrita delta en la matriz martensítica de este tipo de aceros ejerce una influencia negativa en la tenacidad y en la resistencia al creep [4].

Actualmente, se han desarrollado nuevos consumibles que permiten obtener un metal de soldadura de características similares a las de los aceros CSEF. Para soldadura por FCAW (flux cored arc welding) se han diseñado alambres tubulares del sistema 9Cr-Mo en los que se ha reemplazado parte del contenido de Mo por W. Esto ha permitido aumentar la resistencia al creep ya que los carburos de W son más estables que los de Mo [5]. Para la soldadura de estos aceros se recomienda el uso de consumibles conocidos como "matching filler metals". El término matching no se relaciona directamente con la composición química del metal de aporte sino más bien con las propiedades mecánicas. Por lo tanto, el desarrollo de nuevos consumibles tiene como objetivo lograr un metal de soldadura que iguale las propiedades del metal base utilizado.-

En este trabajo se estudió el comportamiento de la junta soldada de tubos de acero $9 \mathrm{Cr} 1 \mathrm{Mo}$, con dos alambres tubulares que depositan aceros $9 \mathrm{Cr} 1 \mathrm{Mo} \mathrm{y} 9 \mathrm{Cr} 0,5 \mathrm{MoWC}$ respectivamente, analizándose la evolución microestructural y las propiedades de tracción y dureza, en esta primera etapa.

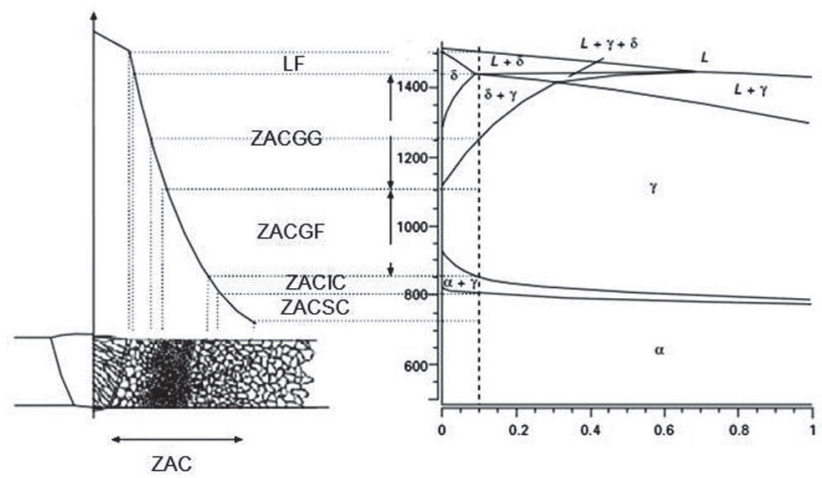

Figura 1. Esquema de las subzonas de la ZAC de acuerdo con el diagrama de equilibrio calculado para aceros del tipo P91

[4].

\section{Materiales y métodos}

\subsection{Materiales utilizados}

Tabla 1a. Composición química del metal base

\begin{tabular}{|c|c|c|c|c|c|c|c|c|c|c|c|c|c|}
\hline $\mathrm{C}$ & $\mathrm{Mn}$ & $\mathrm{Si}$ & $\mathrm{P}$ & $\mathrm{S}$ & $\mathrm{Cr}$ & $\mathrm{Ni}$ & $\mathrm{Cu}$ & $\mathrm{Mo}$ & $\mathrm{Al}$ & $\mathrm{Co}$ & $\mathrm{Nb}$ & $\mathrm{Ti}$ & $\mathrm{V}$ \\
\hline 0,107 & 0,44 & 0,32 & 0,030 & 0,009 & 9,26 & 0,173 & 0,036 & 0,86 & 0,004 & 0,027 & 0,088 & 0,002 & 0,21 \\
\hline
\end{tabular}

Tabla 1b. Propiedades mecánicas del metal base

\begin{tabular}{|c|c|c|c|}
\hline RT $(\mathrm{MPa})$ & LF0,2 $(\mathrm{MPa})$ & A $(\%)$ & HV \\
\hline 705 & 533 & 44 & 222 \\
\hline
\end{tabular}

S/certificado de calidad del acero 
Mabel Ramini, Estela Surian, María José Castillo y Mónica Zalazar

Tabla 2. Composición química del metal de aporte puro

\begin{tabular}{|c|c|c|c|c|c|c|c|c|c|c|c|c|c|c|c|}
\hline \multirow{2}{*}{ alambres } & \multicolumn{15}{|c|}{ elementos (\%) } \\
\hline & $\mathrm{C}$ & $\mathrm{Mn}$ & $\mathrm{Si}$ & $\mathrm{P}$ & $\mathrm{S}$ & $\mathrm{Cr}$ & $\mathrm{Ni}$ & $\mathrm{Cu}$ & Mo & $\mathrm{Al}$ & Co & $\mathrm{Nb}$ & $\mathrm{Ti}$ & $\mathrm{V}$ & $\mathrm{W}$ \\
\hline TuMo & 0,103 & 0,94 & 0,38 & 0,034 & 0,011 & 9,92 & 0,48 & 0,085 & 0,93 & 0,003 & 0,024 & 0,046 & 0,033 & 0,25 & - \\
\hline TuMoW & 0,107 & 0,81 & 0,40 & 0,037 & 0,015 & 9,64 & 0,51 & 0,037 & 0,47 & 0,003 & 1,2 & 0,035 & 0,037 & 0,22 & 1,67 \\
\hline
\end{tabular}

De este modo se obtuvieron los valores Ms para cada consumible y de las temperaturas Tp y Te que se muestran en la Tabla 3.

Tabla 3. Valores calculados según ecuaciones (1) y (2)

\begin{tabular}{|c|c|c|c|}
\hline Probetas & \multicolumn{3}{|c|}{ temperaturas en ${ }^{\circ} \mathrm{C}$} \\
\hline & Ms $\left({ }^{\circ} \mathrm{C}\right)$ & Tp y Te $\left({ }^{\circ} \mathrm{C}\right)$ calculadas & Tp y Te $\left({ }^{\circ} \mathrm{C}\right)$ utilizadas \\
\hline TuMo & 361 & 281 & 260 \\
\hline TuMoW & 343 & 263 & 260 \\
\hline
\end{tabular}

TuMo y TuMoW son probetas de metal de aporte puro de los consumibles

Se utilizó un tubo de acero ASTM A335 GrP91 normalizado y revenido, aleado al Cr-Mo de 28,5 mm de espesor y 350 $\mathrm{mm}$ de diámetro. La condición de suministro fue normalizado: $10 \mathrm{~min}$ a $1050{ }^{\circ} \mathrm{C}$ y recocido: $45 \mathrm{~min}$ a $785{ }^{\circ} \mathrm{C}$. La Tabla $1 \mathrm{a}$ muestra la composición química del metal base y la Tabla $1 \mathrm{~b}$ las propiedades mecánicas. Las soldaduras se realizaron con dos alambres tubulares AWS A5.29/A5.29M:2010 E91T1-B9M y E91T1-G [6], uno tradicional (TuMo) y otro con agregado de W y Co y reducción de Mo (TuMoW).

\subsection{Soldadura de las probetas circunferenciales}

Con cada alambre se soldó una probeta circunferencial mediante el proceso FCAW: PMo y PMoW.

Con el fin de permitir la transformación martensítica en el enfriamiento de cada cordón de la soldadura multipasadas, se eligieron temperaturas de precalentamiento (Tp) y de entrepasadas (Te) inferiores a la temperatura de comienzo de la transformación martensítica (Ms). Para calcular dichas temperaturas se determinó la composición química de los metales de aporte puro [6] de los consumibles utilizados, TuMo y TuMoW, Tabla 2, utilizando las siguientes ecuaciones [7]:

$$
\begin{aligned}
& M s=454-210 C+\frac{4,2}{C}-27 N i-7,8 M n-9,5(C r+M o \\
& +V+W+1,5 S i)-21 C u
\end{aligned}
$$

$$
T p \text { y } T e=(M s-90) \pm 10
$$

Se seleccionó una temperatura de precalentamiento y entrepasadas de $260^{\circ} \mathrm{C}$ para ambos consumibles, con el fin de mantener constantes las condiciones de soldadura. Los parámetros utilizados para realizar las soldaduras circunferenciales fueron: tensión de arco $25 \mathrm{~V}$, intensidad de corriente $155 \mathrm{~A}$ y velocidad de avance $2,6 \mathrm{~mm} / \mathrm{s}$, lo que generó un calor aportado promedio de $1,49 \mathrm{~kJ} / \mathrm{mm}$. Se soldaron cinco capas y 18 cordones, con protección gaseosa de $80 \% \mathrm{Ar} / 20 \% \mathrm{CO}_{2}$ fijando un caudal de 20 $\mathrm{L} / \mathrm{min}$. La preparación de junta correspondió a la presentada en la Figura 2. Una vez realizadas las soldaduras, se enfriaron lentamente al aire y se les realizó un PWHT a $760^{\circ} \mathrm{C}$ durante 4 h (T1) y 2 h (T2).

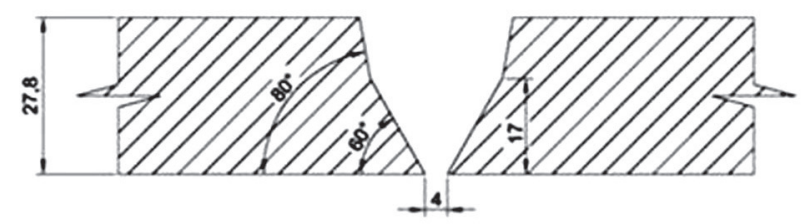

Figura 2. Esquema de la junta soldada (dimensiones en $\mathrm{mm}$ ).

\subsection{Análisis químico}

Mediante espectroscopía de emisión se analizaron las probetas de metal base, de aporte puro y de metal de soldadura. Las probetas para el análisis del metal de aporte puro se prepararon según la norma AWS A5.29/A5.29M:2010 [6] y las de metal de soldadura fueron analizadas sobre las caras superiores de los cordones.

\subsection{Análisis metalográfico y microdureza}

De cada una de las probetas soldadas se prepararon cortes transversales mediante desbaste, pulido y ataque con dos reactivos diferentes: Vilella y Beraha, a fin de analizar la microestructura mediante microscopía óptica (MO) y electrónica de barrido (MEB). El reactivo Beraha, que es un ataque color, permite diferenciar las regiones presentes y los tamaños de grano asociados en la macro y la micrografía y resultó adecuado para MO; el reactivo Villela es adecuado para MO y MEB y pone en relieve los carburos, principalmente.

Se realizaron barridos de microdureza Vickers con $1 \mathrm{~kg}$ de carga, sobre un corte transversal de la junta soldada tomando la 
línea media del metal de soldadura en dirección vertical y una línea horizontal cubriendo desde el MS al MB. Estos perfiles de dureza se relacionaron con cada una de las zonas y subzonas previamente identificadas.

\subsection{Ensayos de tracción}

Las probetas para el ensayo de tracción transversal se extrajeron según indica la Figura 3. El ensayo se realizó según la norma ASTM E8 / E8M - 09 [8].

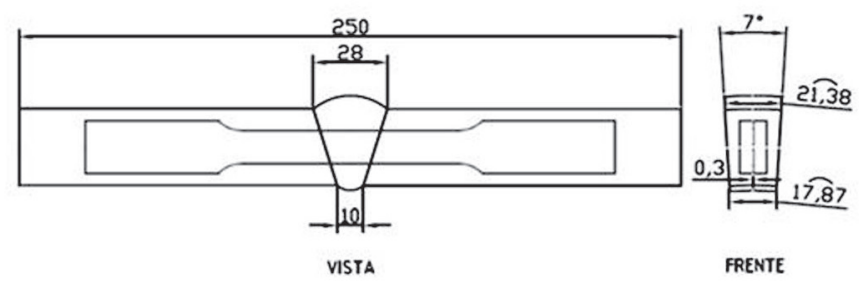

Figura 3. Esquema de extracción de la muestra

\section{Resultados y discusión}

\subsection{Caracterización del metal base}

El MB se caracterizó mediante análisis químico y microscopía óptica. En la Tabla 1a se presenta la composición química correspondiente y en la Figura 4 la microestructura del acero utilizado. En esta fotomicrografía se observa una estructura de martensita revenida y carburos, producto del proceso de fabricación del tubo.

\subsection{Estudios de la junta soldada}

En la Tabla 4 se presentan los resultados del análisis químico realizado en el MS de las probetas soldadas con cada uno de los alambres. Se observa que el agregado de $\mathrm{W}$ al alambre con el que se soldó la probeta PMoW fue compensado con una disminución del contenido de Mo hasta aproximadamente la mitad, el agregado de Co y una pequeña reducción en el contenido de $\mathrm{Cr}$. Esta diferencia en la composición química de los alambres se ha traducido en una diferencia en los valores de $\mathrm{Cr}$ equivalente (Creq), de manera que en la probeta PMo se superó el valor de 10 que es considerado crítico para la presencia de ferrita delta [9], mientras que en la probeta PMoW el Creq se mantuvo por debajo de este valor.

Las Figuras 5 a y 5 b muestran la microestructura observada en el MO, del MS de cada una de las probetas correspondientes al PWHT T1: en ambos casos se observa una estructura de martensita revenida. En las fotomicrografías tomadas en el MEB de las Figuras 5c y 5d se pueden observar los precipitados presentes en los bordes de grano.

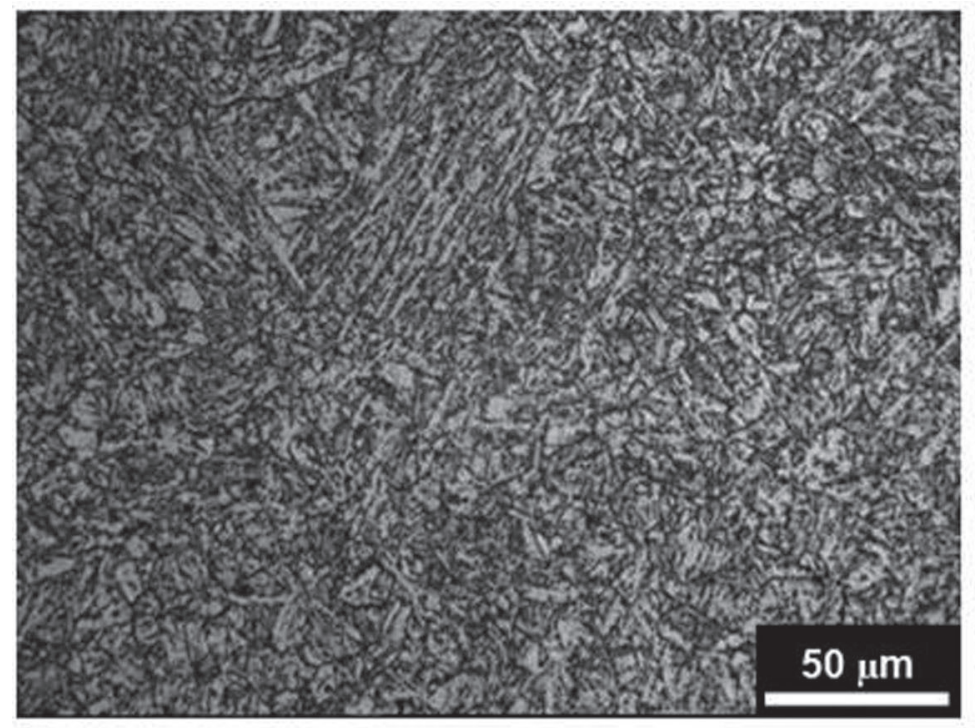

(A)

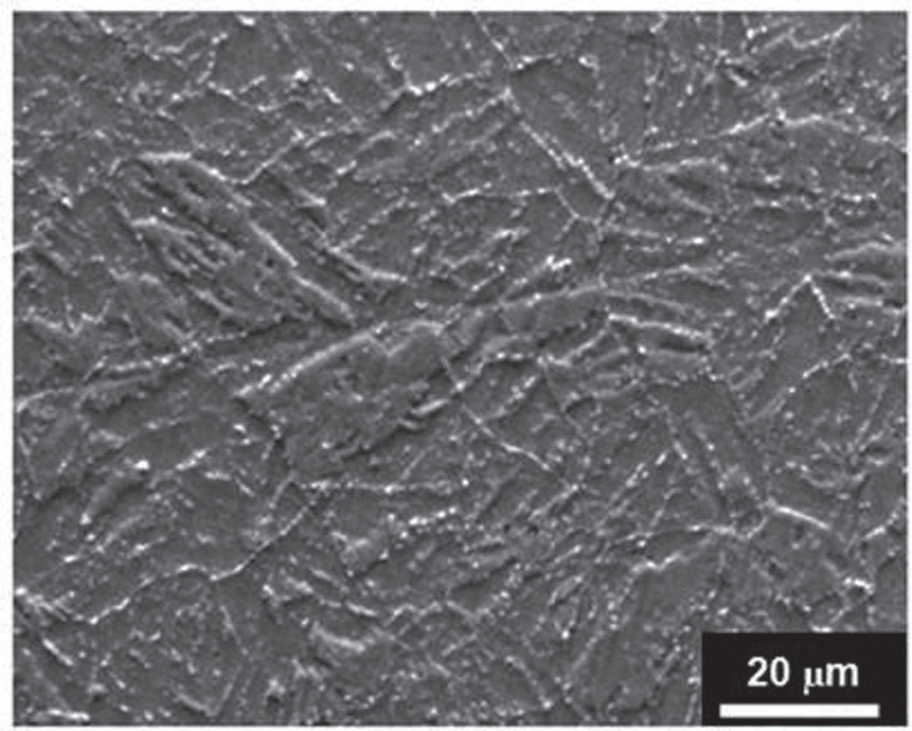

(B)

Figura 4. Microestructura del acero base: A: MO, B: MEB

Tabla 4. Composición química del MS

\begin{tabular}{|c|c|c|c|c|c|c|c|c|c|c|c|c|c|c|c|c|}
\hline \multirow{2}{*}{ probetas } & \multicolumn{16}{|c|}{ elementos $(\%)$} \\
\hline & $\mathrm{C}$ & $\mathrm{Mn}$ & $\mathrm{Si}$ & $\mathrm{P}$ & $\mathrm{S}$ & $\mathrm{Cr}$ & $\mathrm{Ni}$ & $\mathrm{Cu}$ & Mo & $\mathrm{Al}$ & $\mathrm{Co}$ & $\mathrm{Nb}$ & $\mathrm{Ti}$ & V & W & Creq \\
\hline PMo & 0,121 & 0,98 & 0,40 & 0,034 & 0,012 & 9,89 & 0,49 & 0,08 & 1,04 & 0,004 & 0,02 & 0,05 & 0,036 & 0,26 & & 11,0 \\
\hline
\end{tabular}




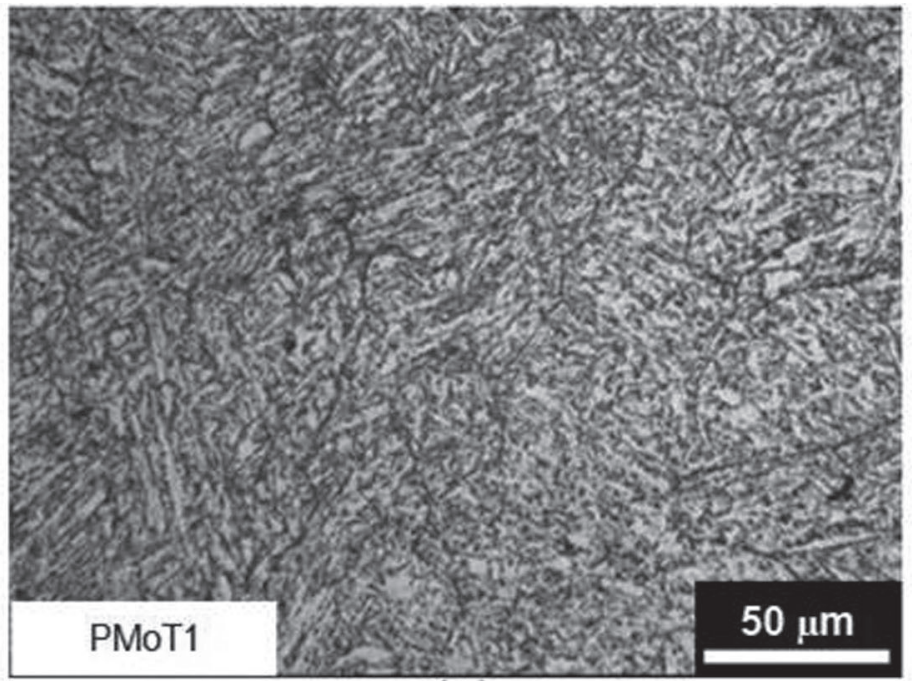

(A)

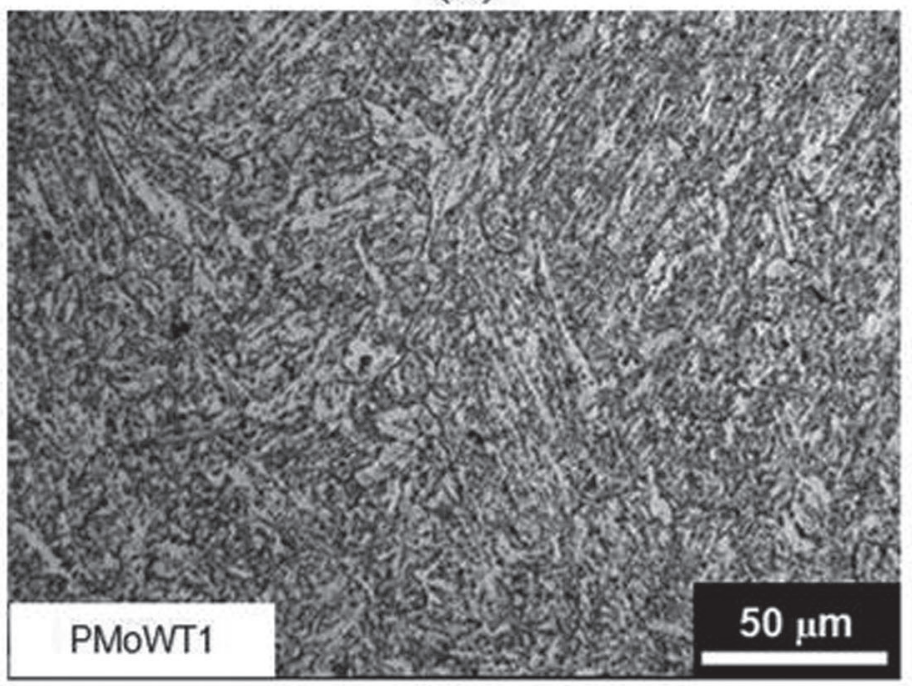

(C)

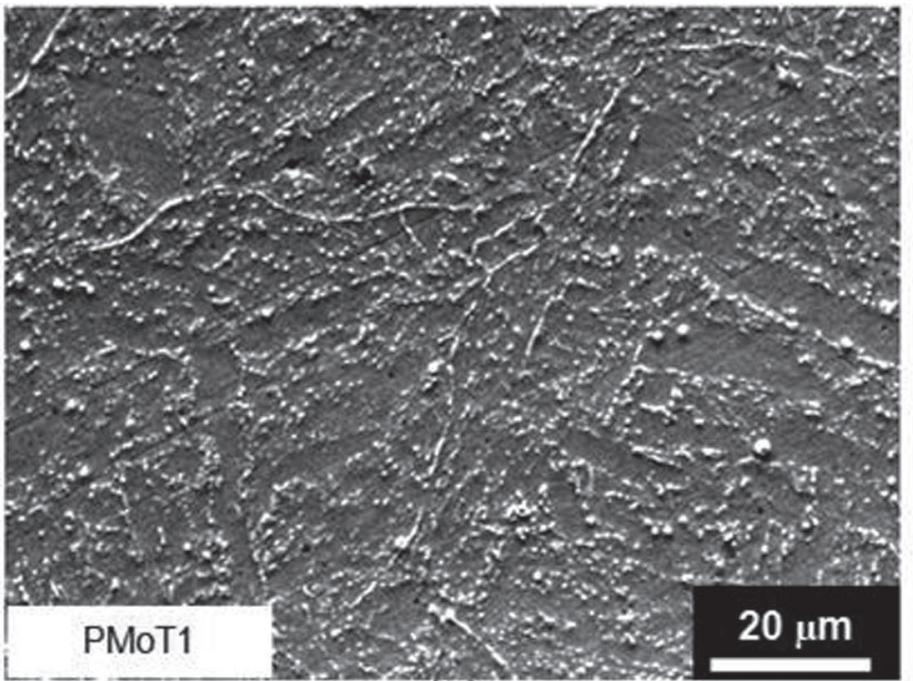

(B)

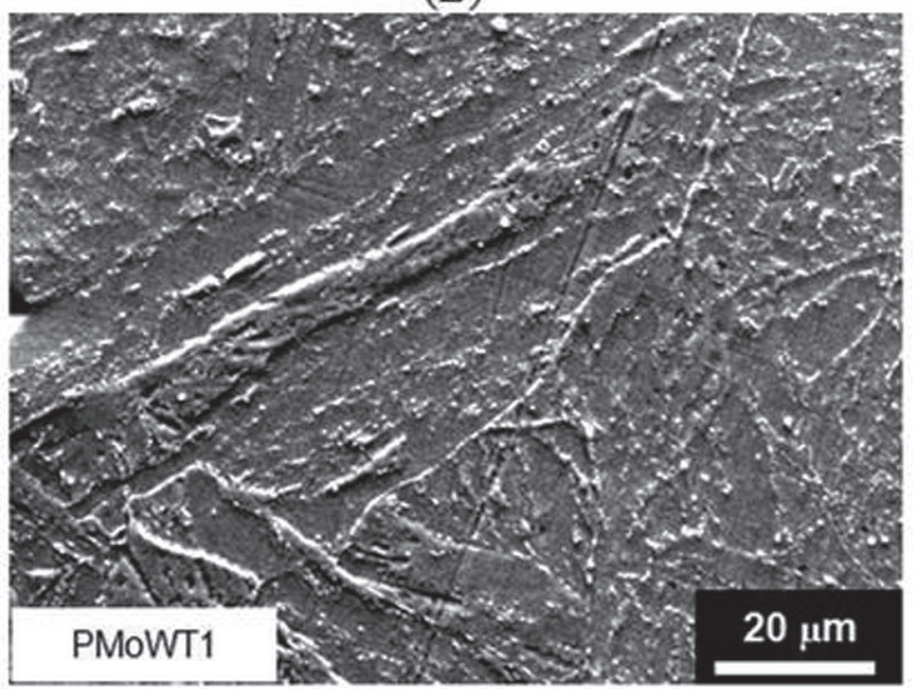

(D)

Figura 5. Microestructura del MS: A y C al MO: B y D al MEB, correspondientes al PWHT T1
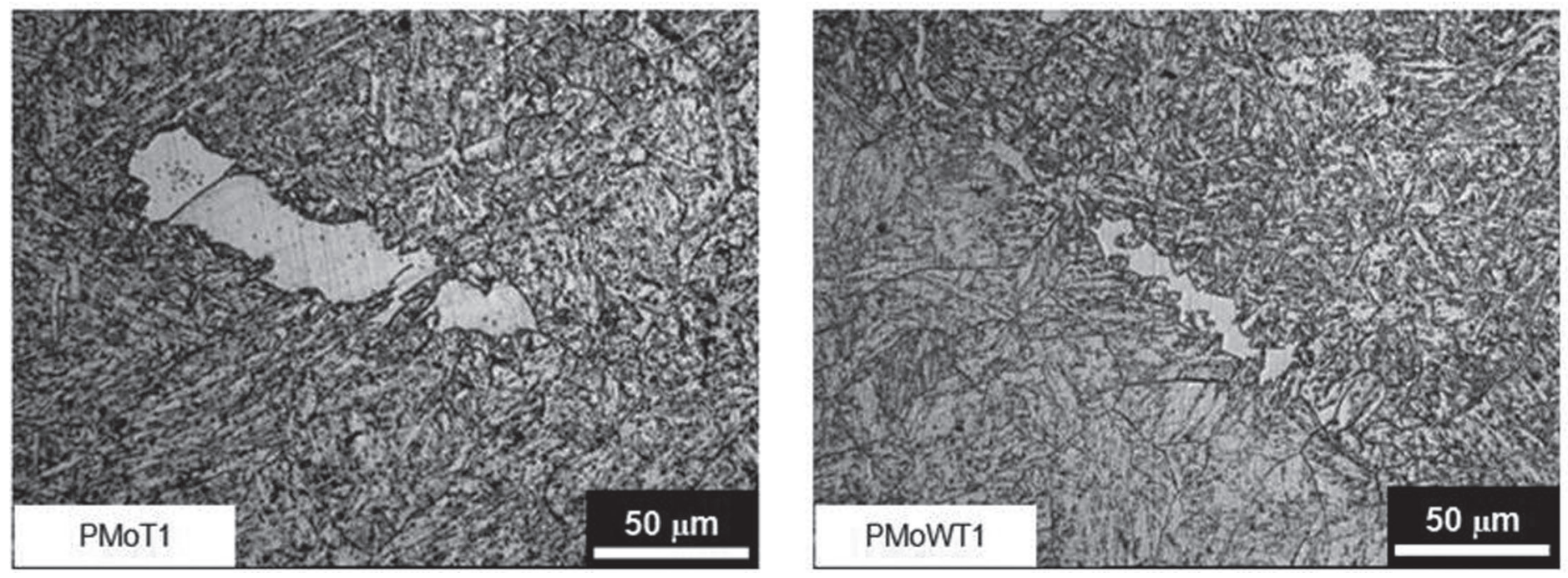

Figura 6. Ferrita delta en bloque 


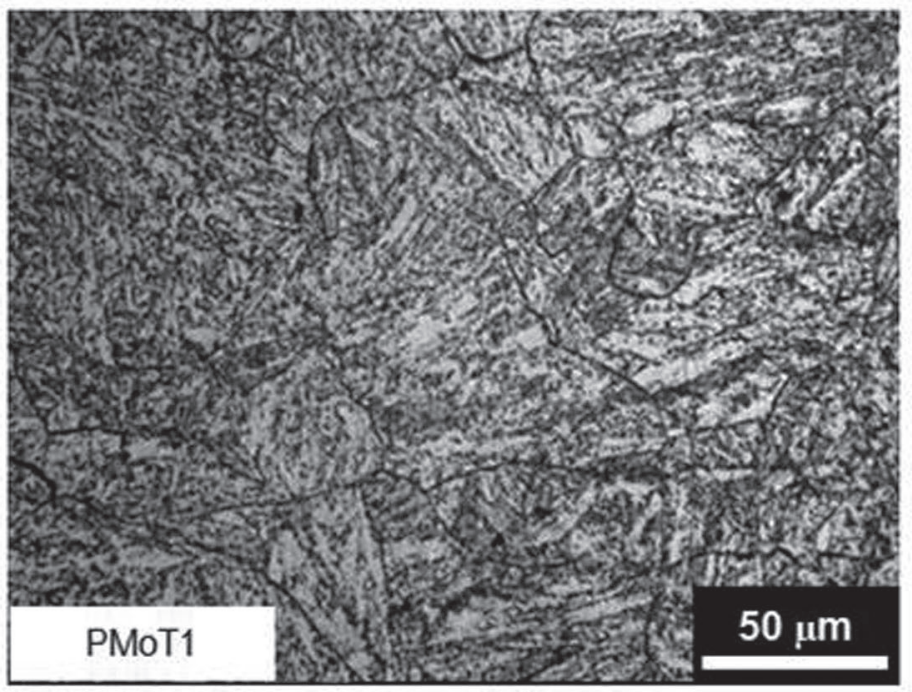

(A)

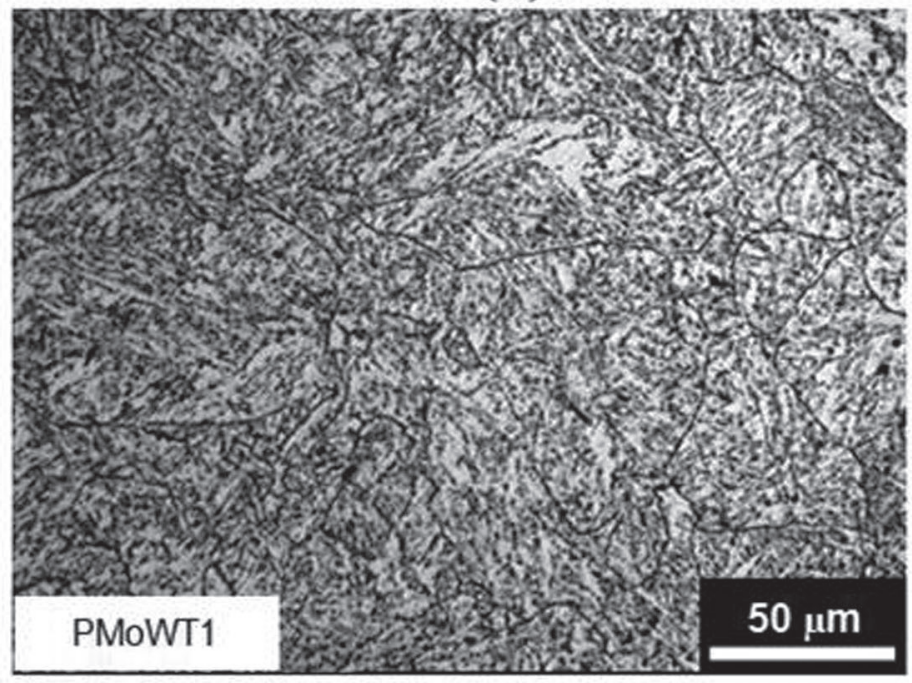

(C)

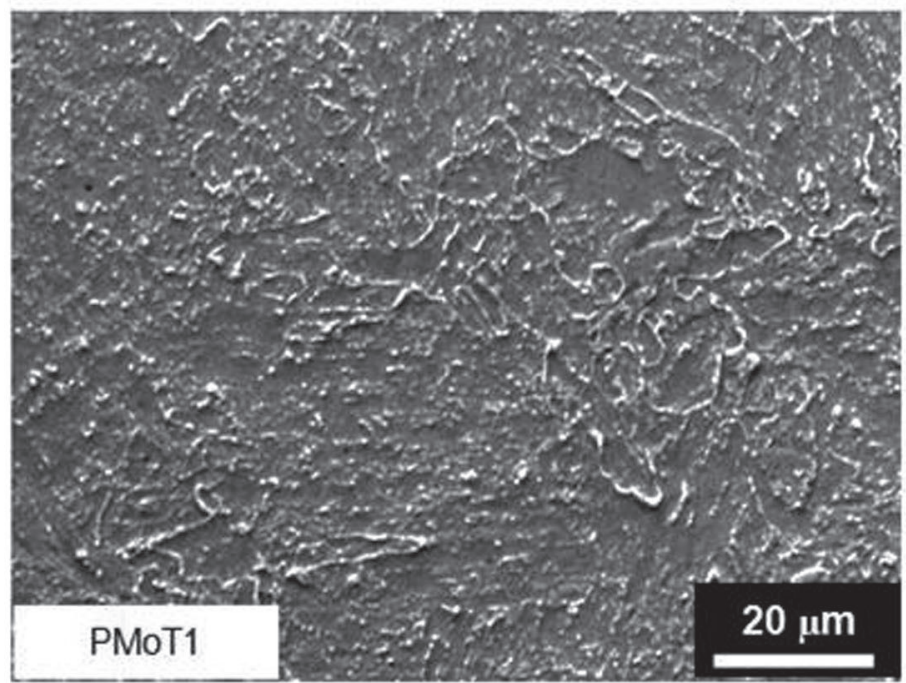

(B)

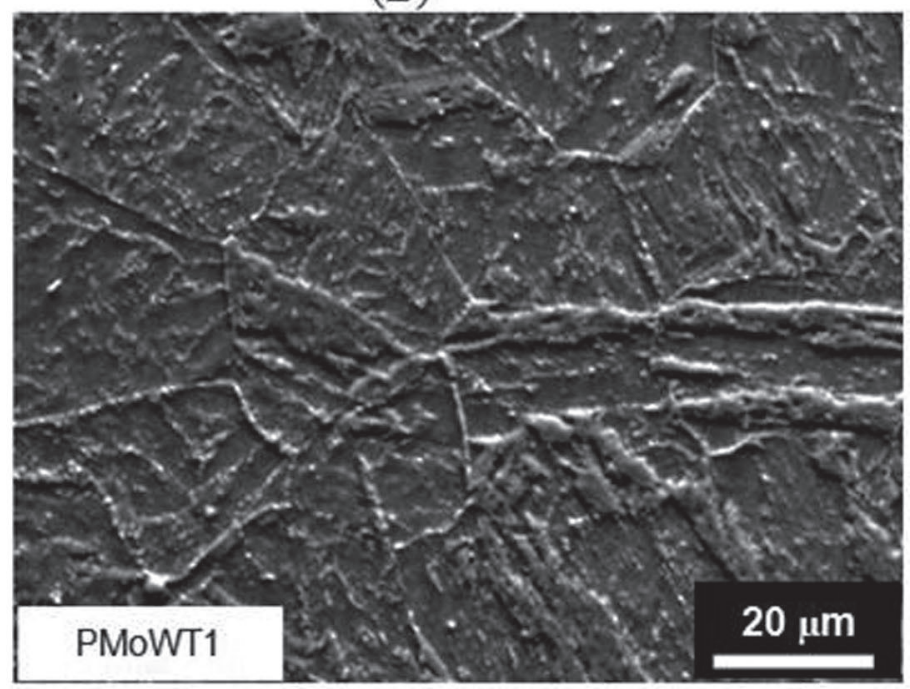

(D)

Figura 7. Microestructura de la ZAC GG. A y C: MO; B y D: MEB correspondientes al PWHT T1

En algunas regiones aisladas de ambas probetas se observaron bloques de ferrita delta, (Figura 6). Los bloques encontrados en la probeta PMoW fueron de menor tamaño y asociados a la línea de fusión. La presencia de ferrita delta en zonas cercanas a la línea de fusión también ha sido observada por otros autores [10]. Una velocidad de enfriamiento elevada en esta zona retiene la ferrita generada a altas temperaturas sin transformar.

La Figura 7 muestra las microestructuras de la ZACGG de ambas probetas correspondientes al PWHT T1 analizadas en el MO y el MEB y la Figura 8 presenta las microestructuras de la ZACGF: como era de esperar, no se detectaron diferencias cualitativas entre las probetas en las observaciones realizadas, dado que los parámetros de soldadura son similares y el MB el mismo.

En las microestructuras analizadas en el MO de las probetas correspondientes al PWHT T2 no se observan diferencias relevantes entre los dos tratamientos térmicos a este nivel de observación.
En la Tabla 5 se presentan los promedios de dureza de las diferentes zonas analizadas. Se observó mayor dureza en las probetas soldadas con el alambre aleado con W, para ambos tratamientos. Un leve aumento de dureza en el MS se manifestó cuando disminuyó el tiempo de mantenimiento a temperatura con el PWHT T2. En la ZAC no se observaron variaciones apreciables para ninguno de los casos analizados.

Tabla 5: Valores promedio de dureza HV1 medidos en los barridos de dureza realizados.

\begin{tabular}{|c|c|c|c|c|}
\hline probetas & MS $(\mathrm{V})$ & MS $(\mathrm{H})$ & ZAC & MB \\
\hline PMoT1 & 239 & 244 & 215 & 210 \\
\hline PMoWT1 & 252 & 257 & 211 & 211 \\
\hline PMoT2 & 247 & 248 & 213 & 210 \\
\hline PMoWT2 & 254 & 257 & 212 & 210 \\
\hline
\end{tabular}

$M S(V)$ : barrido vertical. $M S(H)$ : barrido horizontal 


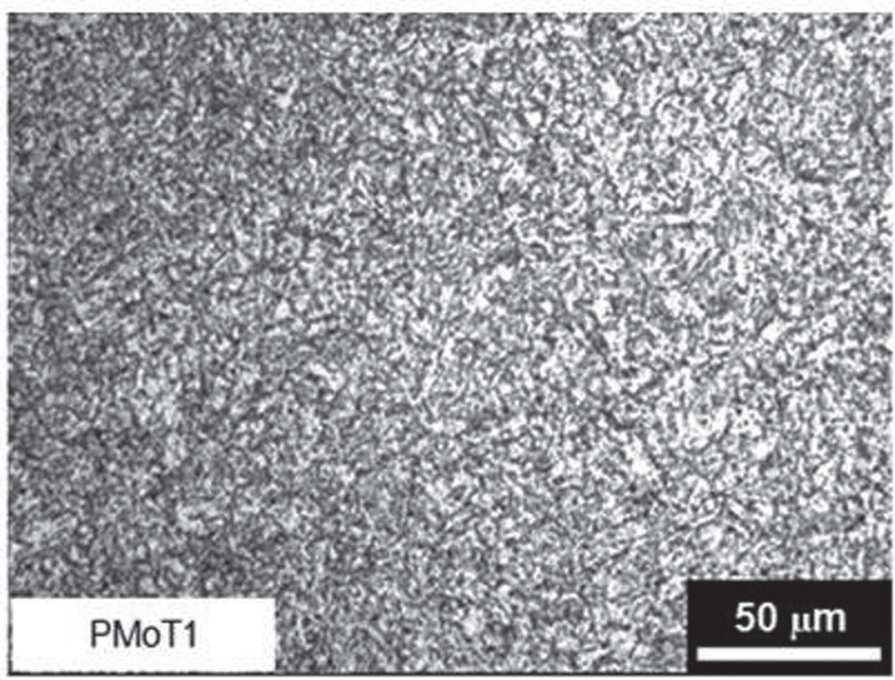

(A)

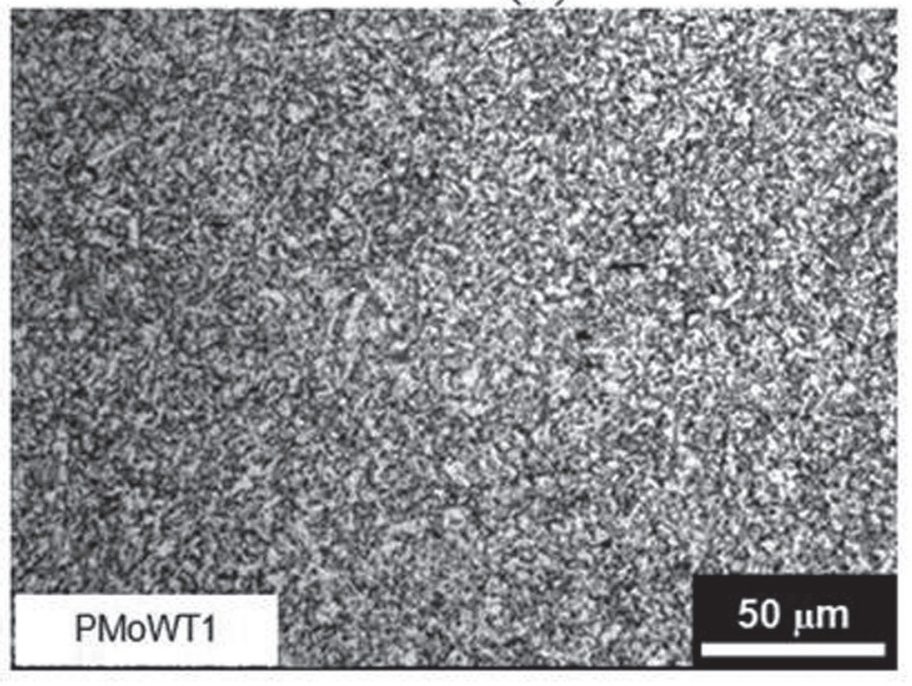

(C)

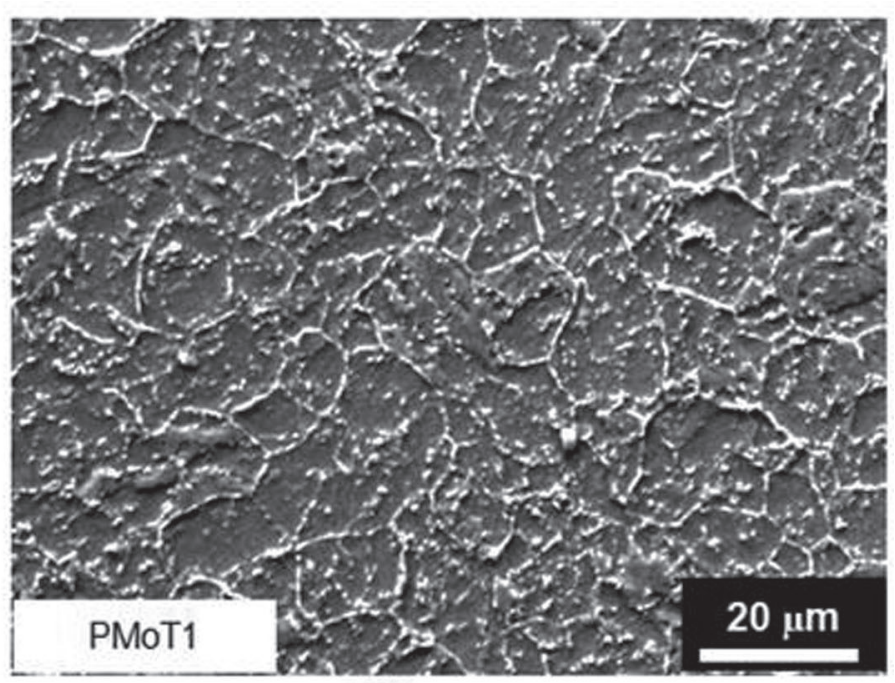

(B)

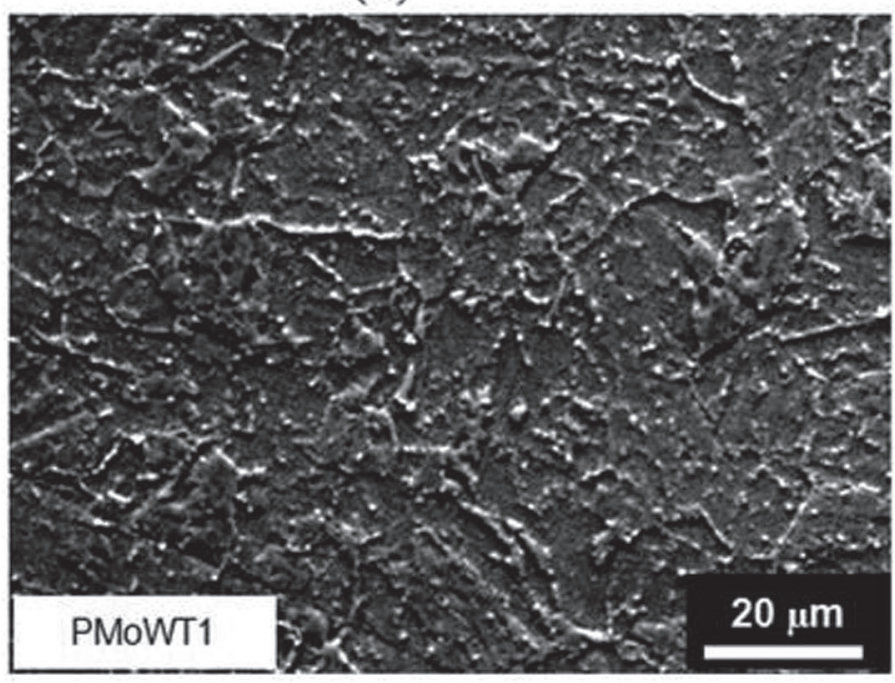

(D)

Figura 8. Microestructura de la ZAC GF. A y C: MO; B y D: MEB correspondientes al PWHT T1

En la Figura 9 se muestran los resultados de los barridos de microdureza correspondientes a los dos cupones soldados con los dos tratamientos térmicos. En todos los casos se observaron valores máximos de dureza en la zona correspondiente al MS y una disminución de la misma en la ZAC desde la zona de GG a la de GF, obteniéndose valores mínimos en la zona IC, inferiores a los correspondientes al MB. Los perfiles de dureza realizados a lo ancho de la ZAC permiten analizar los cambios ocurridos en la microestructura del MB debido al ciclo térmico de la soldadura. Se observó una reducción gradual de la dureza desde la región del MS, mientras que la microestructura, analizada por MO y MEB sólo mostró un cambio en el tamaño de grano austenítico primario. (Figuras 7 y 8). En la ZACGG se soportado las mayores temperaturas, cercanas a la temperatura de fusión; de esta manera el material se austenitizó (Figura 1) y los carburos se disolvieron dejando libre el movimiento de borde de grano. Después del enfriamiento, en la condición como soldado se obtuvo una estructura martensítica, luego revenida en el PWHT. La ZACGG mostró la mayor dureza de la zona afectada por el calor, que generalmente está relacionada con bajos valores de tenacidad [4]. En esta zona los carburos pueden haber sido disueltos por efecto del ciclo térmico de la soldadura y esta disolución haber aportado elementos de aleación que endurecieron la matriz por solución sólida. [11]. Por otro lado, luego del PWHT, pudo haber ocurrido una reprecipitación de carburos que también justificaría dichos valores de dureza.

A medida que aumenta la distancia desde el MS las temperaturas alcanzadas son menores, de manera que en la ZACGF apenas es superada la temperatura de austenización y los precipitados no se disuelven completamente frenando el crecimiento de grano [2]. Luego del enfriamiento y posterior PWHT se obtuvo una estructura refinada de martensita revenida de una dureza menor a la de la ZACGG.

Cuando la temperatura máxima alcanzada llega a la zona 

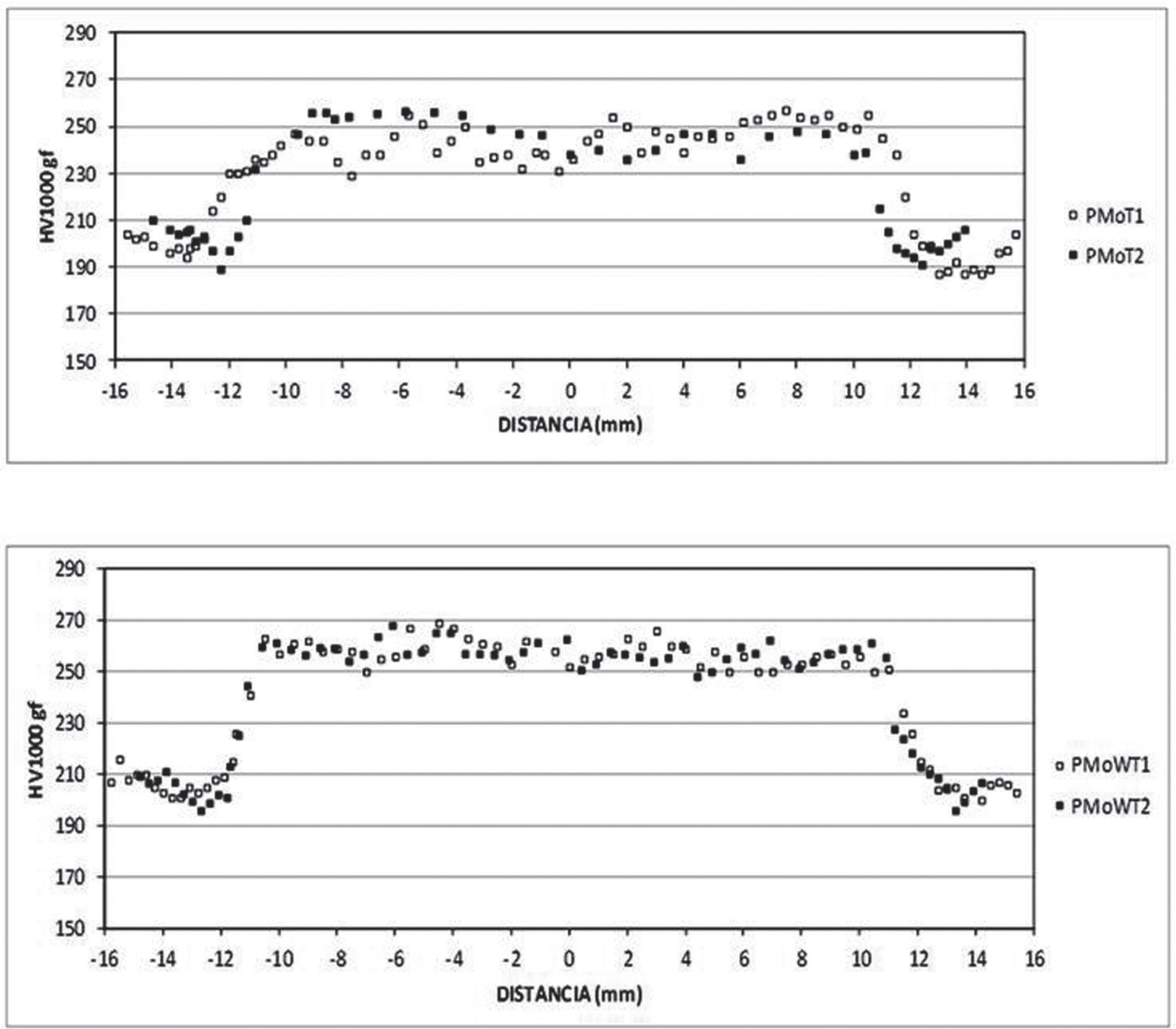

Figura 9. Perfiles de microdureza de las juntas soldadas

intercrítica $\left(\mathrm{Ac}_{1}<\mathrm{T}<\mathrm{Ac}_{3}\right)$ se produce la transformación de la ZACIC, en la cual los precipitados no se disuelven pero pueden crecer y la estructura puede resultar con ferrita proeutectoide [2], producto de la transformación en la zona dúplex (austenita +ferrita) (Figura 1). En esta zona se obtuvieron las menores durezas de la ZAC, tanto para las probetas PMo como para las $\mathrm{PMoW}$, siendo aún menores que las del metal base; este fenómeno se presentó para los dos PWHT con una leve tendencia a menores valores de dureza para el T2.

Los resultados del ensayo de tracción transversal se muestran en la Tabla 6. Puede observarse que los valores obtenidos con la probeta PMo fueron menores que los de PMoW, en coherencia con los valores de dureza. La presencia de $\mathrm{W}$ probablemente fue responsable del incremento de estas propiedades. El menor tiempo de tratamiento térmico (T2) permitió obtener mayores valores de resistencia a la tracción.

Tabla 6: Valores de tracción transversal de la junta soldada

\begin{tabular}{|c|c|c|c|c|}
\hline probetas & $\begin{array}{c}\text { RT } \\
(\mathrm{MPa})\end{array}$ & $\begin{array}{c}\text { LF0,2 } \\
(\mathrm{MPa})\end{array}$ & A (\%) & RA (\%) \\
\hline PMoT1 & 715 & 553 & 18 & 60 \\
\hline PMoWT1 & 722 & 561 & 17 & 59 \\
\hline PMoT2 & 734 & 569 & 11 & 53 \\
\hline PMoWT2 & 746 & 590 & 16 & 59 \\
\hline
\end{tabular}

En todos los casos se superaron los valores de RT y LF del certificado de calidad del acero. 


\section{Conclusiones}

Del análisis de las juntas soldadas de tubos de acero 9Cr1Mo con dos alambres tubulares que depositan aceros $9 \mathrm{Cr} 1 \mathrm{Mo} \mathrm{y}$ $9 \mathrm{Cr} 0,5 \mathrm{MoWCo}$, tratadas térmicamente a $760^{\circ} \mathrm{C}$ durante 2 y 4 horas se obtuvieron las siguientes conclusiones:

- En todos los casos la microestructura del MS consistió en martensita revenida y ferrita delta retenida.

- Los perfiles de dureza mostraron igual comportamiento en todos los casos: mayores durezas en el MS y una caída sostenida hasta llegar a la ZACIC donde se observó un ablandamiento del MB.

- Los valores de dureza fueron mayores en la probeta correspondiente al alambre con agregado de W y Co, especialmente en el MS.

- El efecto del agregado de W y Co en las propiedades de tracción se tradujo en un aumento de la RT y LF para ambos PWHT.

- Con menores tiempos de PWHT se obtuvieron valores más altos de resistencia a la tracción y resultados similares en la microestructura y la microdureza, con un leve aumento de esta última propiedad.

\section{Agradecimientos}

Los autores agradecen a Lincoln Argentina por la donación de los consumibles utilizados, a Conarco-ESAB, Argentina por la realización de los análisis químicos y a APUEMFI por el soporte financiero.

\section{Referencias bibliográficas}

[1] W. F. NEWELL Jr. Welding and Postweld Heat Treatment of P91 Steels. Welding Journal, v 89, N4, pp. 33-36. 2010.

[2] VIJAYALAKSHMI, M. et al. Microstructural Zones in the Primary Solidification Structure of Weldment of 9Cr-1Mo Steel. Metallurgical and Materials Transactions A. v 30A, pp. 161-174, January 1999.

[3] SMITH, D. J., WALKER, N. S., KIMMINS, S.T. Type IV creep accumulation and failure in steel welds. International Journal of Pressure Vessels and Piping, v 80 617-627, 2003.

[4] Creep-resistant steels. Libro editado por Fujio Abe, TorstenUlf Kern and R. Viswanathan. Woodhead. Publishing and Maney Publishing, Cambridge, Reino Unido, 2008.

[5] MIYATA K., SAWARAGI, Y. Effect of Mo and W on the Phase Stability of Precipitates in Low Cr Heat Resistant Steels. ISIJ International, v 41, N 3, pp. 281-289, 2001.

[6] A5.29/A5.29M:2010 Specification for Low-Alloy Steel Electrodes for Flux Cored Arc Welding. American Welding Society, Miami, USA, 2010.

[7] L. BÉRES, A. BALOGH, W. IRMER. Welding of Martensitic Creep-Resistant Steels: the relationship between preheat and Ms temperatures was investigated. Welding Journal, v $80 \mathrm{~N}^{\circ} 8$, pp191s-195s, 2001.

[8] ASTM E8/E8M-09 Standard Test Methods for Tension Testing of Metallic Materials. ASTM International, 2009.
[9] M. SIREESHA, S.K. ALBERT, S. SUNDARESAN. Microstructure and mechanical properties of weld fusion zones in modified $9 \mathrm{Cr}-1 \mathrm{Mo}$ steel. Journal of Materials Engineering and Performance, v 10(3), pp 320-330, 2001.

[10] V.T. PAUL et al. Identification of microstructural zones and thermal cycles in a weldment of modified 9Cr-1Mo steel. J Mater Sci v 42 pp.5700-5713, 2007.

[11] G. George et al. On the microstructure-polarization behavior correlation of a $9 \mathrm{Cr}-1 \mathrm{Mo}$ steel weld joint. Journal of Materials Engineering and Performance, v 10(4), pp 460-467, 2001. 